\title{
Thermoelectric Power of Layered Chalcogenides GaTe Crystals
}

\author{
A.A. Al-Ghamdi \\ Physics Department, Faculty of Science, \\ King Abdulaziz University, Jeddah, Saudi Arabia \\ aghamdi90@hotmail.com
}

\begin{abstract}
Thermoelectric power (TEP) measurements of Gallium mono-telluride single crystals have been studied over the temperature range 193-583K. GaTe single crystals grown from melt by the modified Bridgman technique. The results of measurements indicate that the investigated samples turned out to be p-type nature. Investigation of GaTe compound revealed that it has interesting properties. Many physical parameters were determined such as carrier mobilities, effective masses of free charge carriers, diffusion coefficient and diffusion length as well as the relaxation time. The highest value of figure of merit for GaTe permits the practical application as thermoelectric element.
\end{abstract}

Keywords: Chalcogenide, GaTa, Single Crystals, Thermoelectric Power.

\section{Introduction.}

GaTe is a $\mathrm{A}^{\mathrm{III}} \mathrm{B}^{\mathrm{VI}}$ type semiconducting compound like GaSe, GaS and InSe, which crystallize in a layered structure. Mainly, due to the difficulties in growing good quality large size crystals, it is one of the less investigated materials ${ }^{[1]}$. In the case of $\mathrm{GaTe}$, atomic planes are in Te-Ga-Ga-Te sequence, grouped in layers of four atomic planes where portions of each layer are joined together by regions in which the Ga-Ga bonds are approximately at right angles to the usual Ga-Ga bonds ${ }^{[2]}$. The anisotropy arises from the fact that the bonding within the layers is considerably stronger than that perpendicular to them. In GaTe, Van der Waals forces contribute predominantly to the interlayer interaction, while the bonding forces within the layers are primarily ionic-covalent. Gallium- 
Tellurium is a binary alloy system which exhibits anomalous physical and chemical properties ${ }^{[3]}$. Studies undertaken for the establishment of the equilibrium diagram of the gallium tellurium system proved the existence of the eqiuatomic compound GaTe which is formed by eutectic reaction at $1109 \mathrm{~K}$. The monoclinic lattice ${ }^{[4]}$ of GaTe has a space symmetry group $C_{2}^{2}$ where $\mathrm{a}=11.95 \AA \mathrm{b}=4.04 \AA$ and $\mathrm{c}=14.82 \AA$. Most published works presented in the literature are interested in studying the crystal structure ${ }^{[4-6]}$ visible reflection spectra $^{[7]}$, the photoconductivity spectra ${ }^{[8]}$ the anisotropy in the absorption spectrum at the fundamental edge ${ }^{[9]}$ also the electrical conductivity and Hall effect ${ }^{[10,11]}$ photoluminescence characterization ${ }^{[12]}$, Raman spectra ${ }^{[13]}$ memory switching ${ }^{[14]}$ and other physical properties ${ }^{[15]}$. Thermoelectric properties have not been catalogued in the literature. However no information at all was published on thermoelectric power of GaTe as far as we know. In view of this the present work aims to investigate the thermoelectric properties which is essential to the understanding of the material and consequently their application in energy conversion devices.

\section{Experimental Procedures}

\section{a-Sample Preparation}

GaTe single crystal was grown from melt using Bridgman technique in solid state laboratory. Special design was used for crystal growth, which is a part of our program for locally produced equipment for physics research. This design demonstrates that the driving force required to move the loaded silica ampoule is equivalent to the decrease of the water level drained in a special container which in turn indicated the rate of motion. The sample was prepared from highpurity gallium $(6 \mathrm{~N})$ representing $64.656 \%$ and tellurium $(5 \mathrm{~N})$ representing $35.3439 \%$. The principle of the design and details of the process were published previously ${ }^{[16]}$. Samples obtained were single crystals of good quality and were brittles showing a layer structure. The crystal perfection was checked using the $\mathrm{X}$-ray diffraction technique. Diffraction for these materials compared with the index data of the American society for testing materials (ASTM) cards. From these X-ray studies it was evident that the crystals have a high degree of crystallinity indicating that the preparation technique is farily reliable and satisfactory.

\section{b - Experimental Arrangement}

Single crystal samples were prepared by gentely cleavage, from the product ingot with the aid of a razor blade. The length of the sample was chosen to be as short as possible, whereas the cross sectional area was large enough. The surfaces of the sample were flat and parallel. The prepared sample was of a desk 
shape with diameter $9.8 \mathrm{~mm}$ and thickness $3.8 \mathrm{~mm}$. It was necessary to have the temperature equally distributed. Therefore we placed contacts of silver past across the ends of the specimen uniformly. A two part holder was used for making the temperature difference along the crystal in a direction perpendicular to the natural cleavage plane. The ambient temperature was produced by one of them, but the second heater was electrically energized to have the temperature difference. The sample was insulated from the holders by thin sheets of mica. The sample was clamped between the two holders gentely and introduced inside an evacuated working chamber. Two well calibrated thermocouples, made of copper constantan, were used for measuring the sample temperature at each end. The temperature of the crystal was considered to be the average of those at its two ends. The temperature difference was kept small (about 5K). Simultaneous measurements of temperature and potential difference were carried out to increase the accuracy of the measurements. Details about apparatus and experimental method measuring the differential thermoelectric power $(\alpha)$ have been described in previous paper ${ }^{[18]}$. Measurements were carried out, during this investigation in a wide range of temperature extending from 193 up to $583 \mathrm{~K}$.

\section{Results and Discussion}

The variation of the differential thermoelectric power $(\alpha)$ of GaTe-single crystal as a function of temperature is illustrated in Fig. 1. Results and the figure indicate the following points: (1) Our sample shows P-type conductivity. (2) The figure shows that at the beginning of the curve TEP decreases slowly as the temperature rises till reaching $380 \mathrm{~K}$, after which $\alpha$ decreases rapidly reaching its smallest value $85 \mu \mathrm{V} / \mathrm{K}$ at $435 \mathrm{~K}$, this may be due to presence of some crystal defects or trapping centers in the direction of the carrier flow. (3) Above $435 \mathrm{~K}$ with further rise of temperature, $\alpha$ increases rapidly till reaching a value of about $250 \mu \mathrm{V} / \mathrm{K}$ corresponding to $583 \mathrm{~K}$. Such behavior is expected in this intrinsic range where generation of both carriers (electrons and holes) contributed to the increment of $(\alpha)$ value. In the intrinsic region we can apply the following formula ${ }^{[19]}$.

$$
\alpha=-\frac{K}{e}\left[\frac{b-1}{b+1}\left(\frac{\Delta E_{g}}{2 K T}+2\right)+\frac{3}{4} \ln \frac{m_{n}^{*}}{m_{p}^{*}}\right]
$$

Where $\mathrm{K}$ is the Boltzman constant, $b$ is the ratio between the electron and hole mobilities, $m_{n}^{*}$ and $m_{p}^{*}$ are the effective masses of electrons and holes respectively and $\Delta \mathrm{Eg}$ is the energy gap. In accordance with this equation a plot of $\alpha$ as a function of reciprocal of absolute temperature is a straight line as shown in Fig. 2. The measured thermoelectric power in conjuction with the published Hall data ${ }^{[11]}$ is used to calculate the required physical parameters. The 


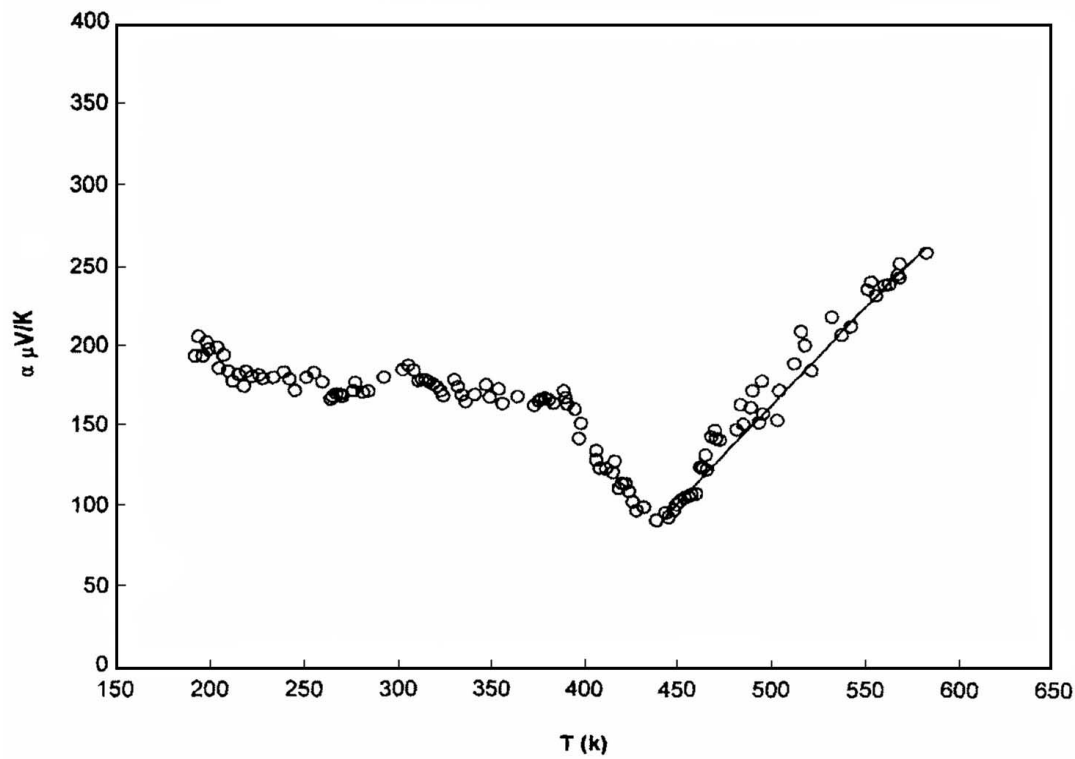

Fig. 1. Temperature dependence of thermoelectric power.

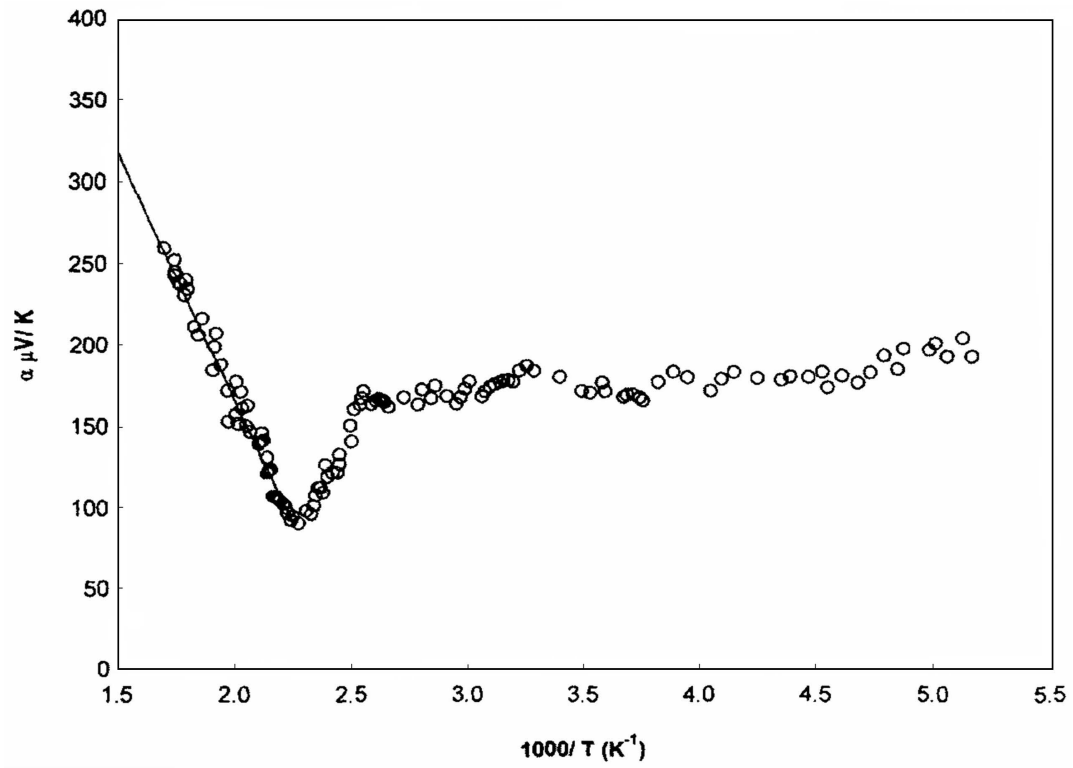

Fig. 2. Relation between $\alpha$ and 1000/ T. 
slope of the linear part of the dependence in the intrinsic region is used to estimate the ratio of electron to hole mobilities. Taking $\Delta \mathrm{E}_{\mathrm{g}}=1.6 \mathrm{eV}$. and assuming that $\frac{m_{n}^{*}}{m_{p}^{*}}$ does not vary with temperature, it can be deduced that $b \frac{\mu_{n}}{\mu_{p}}=2.268$. Since $\mu p 12 \mathrm{~cm}^{2} / \mathrm{V}$.sec, the electron mobility is found to be $27.216 \mathrm{~cm}^{2} / \mathrm{V}$.sec. The ratio between the effective masses of both electrons and holes can be evaluated from the intersection of the curve, and is found to be $\frac{m_{n}^{*}}{m_{p}^{*}}=2.525 \times 10^{-3}$. The diffusion coefficient for majority and minority carriers at room temperature can be estimated and is found to be $D_{n}=0.705$ $\mathrm{cm}^{2} \mathrm{sec}^{-1}$, and $\mathrm{D}_{\mathrm{p}}=0.311 \mathrm{~cm}^{2} \mathrm{sec}^{-1}$. The relation between $\alpha$ and $\mathrm{In} \mathrm{T}$ is illustrated in Fig. 3. At low temperature the value of TEP decreases slowly and gradually with temperature. In the transition region a drop of the value of TEP is observed as the temperature increases. In the intrinsic region the value of $\alpha$ increases with temperature. Calculation of the TEP in the impurity region, yields the following results.

$$
\alpha=\frac{K}{e}\left[2-\ln \frac{p h^{3}}{2\left(2 \pi m_{p}^{*} K T\right)^{3 / 2}}\right]
$$

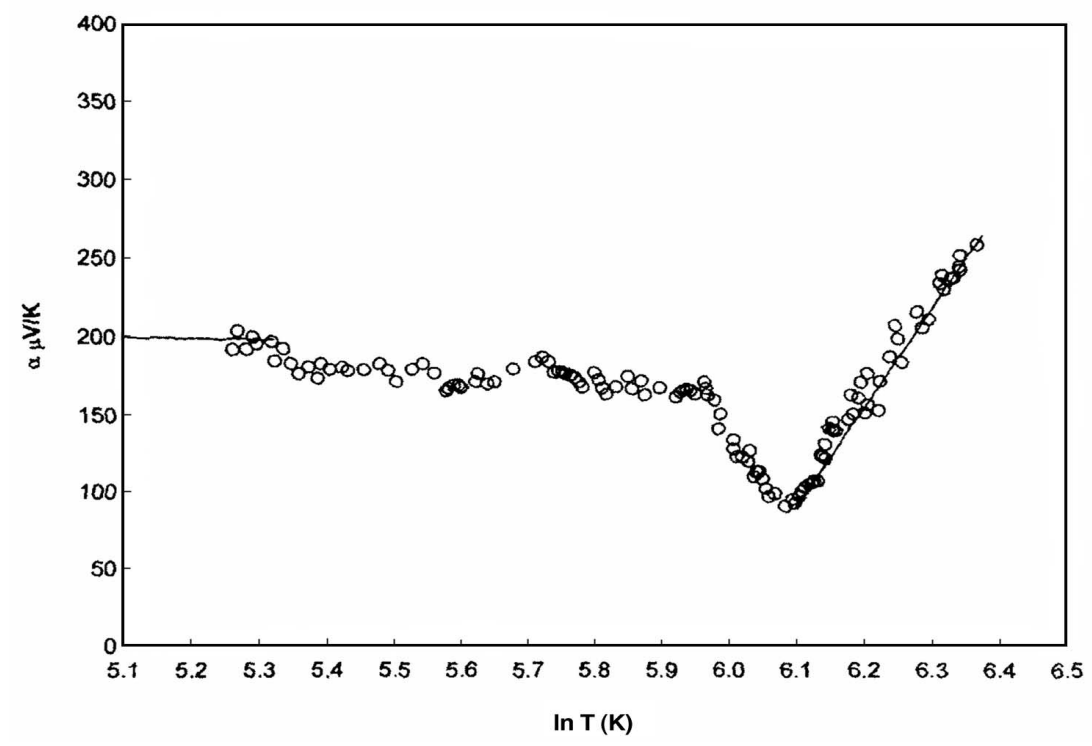

Fig. 3. Relation between $\alpha$ and $\ln T$. 
Where the sign of $\alpha$ is the sign of the charge carrier, $p$ represents the concentration of free charge carriers (hole) and $m_{p}^{*}$ denotes the effective mass of holes. Figure 4 shows the dependence of thermoelectric power on carrier concentration for GaTe sample. As it is shown $\alpha$ decreases linearly with increasing of carrier concentration. Calculation of the effective mass of hole from the intersection of the curve yields consistently the value $m_{p}^{*}=8.109 \times 10^{-29} \mathrm{~kg}$. Combining this value with the above mentioned result for the ratio $m_{n}^{*} / m_{p}^{*}$, one can obtain the effective mass of electrons $m_{n}^{*}=2.047 \times 10^{-31} \mathrm{~kg}$.

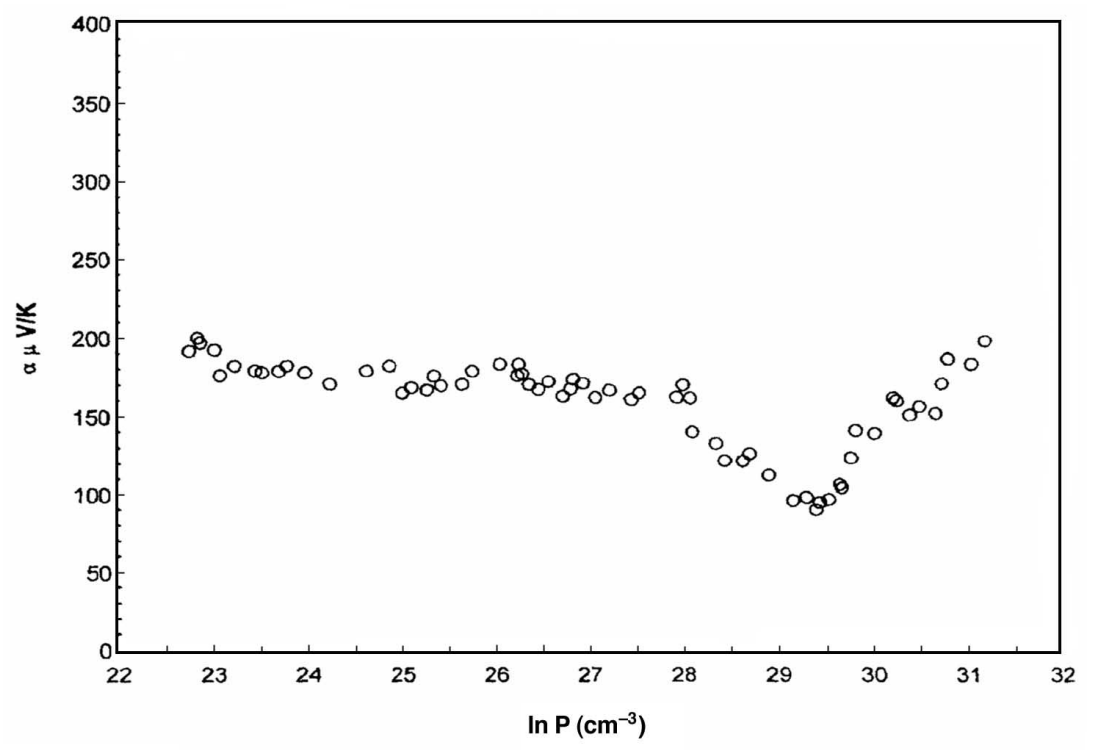

Fig. 4. Relation between $\alpha$ and charge carrier concentration.

We also deduced the effective masses of holes and electrons related to the rest mass, and the value $m_{n}^{*}=98 \mathrm{f} m_{0}$ or holes and $m_{n}^{*}=0.225 m_{\mathrm{o}}$ for electron. Since the effective mass values are available, now the relaxation time for both types of carriers can be determined. The relaxation time for holes, as calculated is $6.08 \times 10^{-16} \mathrm{sec}$ whereas the relaxation time for electrons equals $3.482 \times$ $10^{-18} \mathrm{sec}$. It is noticed that the diffusion constant is inversely proportional to the effective mass of the carriers. This is logical because the hole effective mass is larger than that of the electrons.

The diffusion length can also be established from our data. The diffusion length for holes and electrons are $\mathrm{L}_{\mathrm{p}}=1.375 \times 10^{-8} \mathrm{~cm}$ and $\mathrm{L}_{\mathrm{n}}=1.567 \times 10^{-9}$ $\mathrm{cm}$ respectively. Figure 5 represents the relation between TEP and the electrical conductivity. It is seen from the curve that three regions is noticed. The values 
of $\alpha$ at the first region decreases gradually. At conductivity equal to $1.65 \times$ $10^{-12}(\Omega \mathrm{cm})^{-1}$ a rapid decrease of $\alpha$ is observed. The value of TEP corresponding to the minimum is about $90 \mu \mathrm{V} / \mathrm{K}$ at $\sigma=6.68 \times 10^{12}(\Omega \mathrm{cm})^{-1}$ after which TEP increases rapidly as the electrical conductivity continues to increase. The behavior of the curve between charge carrier concentration and TEP is similar to that of $\alpha$ Vs. $\sigma$. However, those parameters are found to be sufficient to give complete information about the general behavior of the GaTe single crystal. The choice of materials for thermocouples, thermoelectric generation, and, refrigerators is based on the efficiency parameter $\mathrm{Z}$, defined by the relation $\mathrm{Z}=\frac{\alpha^{2} \sigma}{K}$. Where $\alpha$ is the differential thermoelectric power and $\sigma$ is the electrical conductivity and $\mathrm{K}$ is thermal conductivity. However the term figure of merit $(Z)$ is a measure of both performance and efficiency of a certain thermoelectric element. Therefore the main technical problem to obtain a good thermoelectric element is to answer the question, how to promote the figure of merit of the material. The best material to be used as thermoelectric element is that with the highest value of $\mathrm{Z}$.

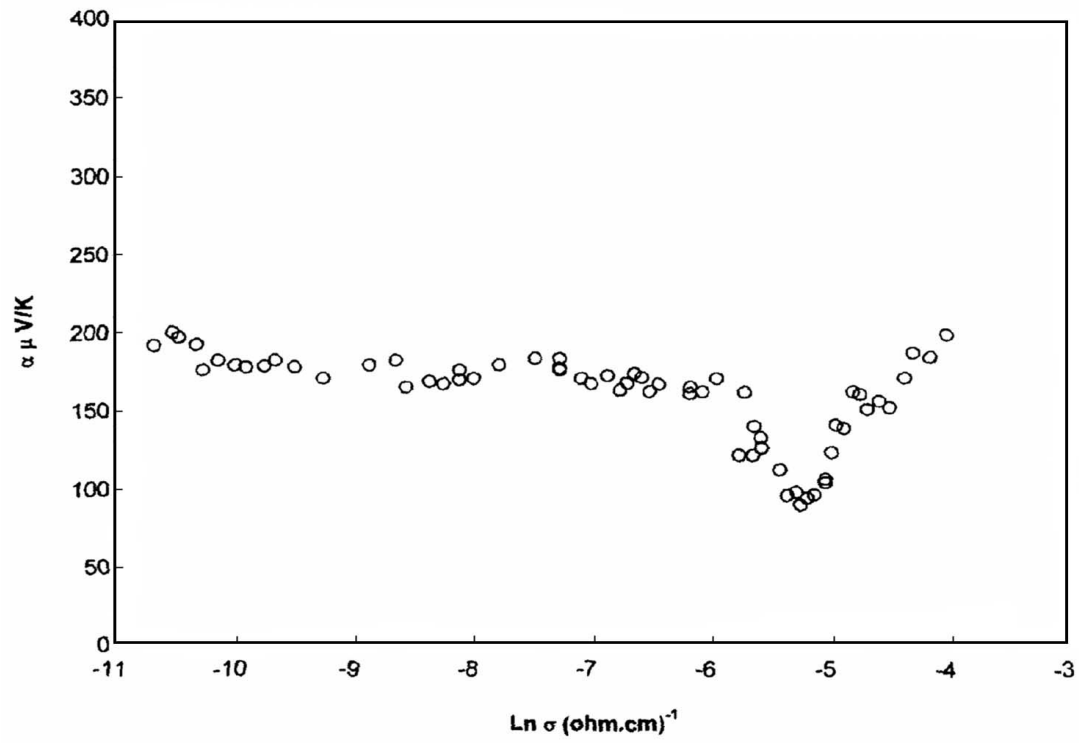

Fig. 5. Variation of $\alpha$ with electrical conductivity.

For our best GaTe samples the figure of merit at $300 \mathrm{~K}$ is $\mathrm{Z}=37.27 \mathrm{~K}^{-1}$. One determines this value by using the published value of $\mathrm{K}^{[20]}$ which is equal to $10.1 \mathrm{WK}^{-1} \mathrm{~m}^{-1}$. The highest value of $\mathrm{Z}$ for GaTe permits the practical application as thermoelectric element. 


\section{References}

[1] Aydinli, A., Gasanly, N.M., Uka, A. and Efeoglu, H., Cryst. Res. Technol., 37: 1303 (2002).

[2] Evans, B.L., Optical Properties of Layer Compounds, Ed. P.A. Lee, D. Reidel Publishing company, Dordrecht- Holland P1 (1976).

[3] Muller, A., Hoger, W., Thomas, E. and Wobst, M., Phys. Stat. Sol. (a), 81: K 97 (1984).

[4] Stubbs, M.F., Scuffle, I.A., Thompson, A.I. and Duhnken, I.M., J. Amer. Chem. Soc., 14: 1441 (1952).

[5] Allakhverdiev, K.R., Gasymov, SH. G., Mamedov, T.G., Nizametinova, M.A. and Sallev, E.Ya., Phys. Semicond., 2: 1 (1983).

[6] Alapini, F., Flahaut, J., Guittard, M., Jaulmes, S. and Julien-Pouzol, M., J. Solid Stat. Chem., 28:309 (1979).

[7] Soboler, V.V., Kramer, V.M. and Kovalyuk, Z.D., J. Appl. Spectroscopy, (USA), 39: 52 (1983).

[8] Kurbatov, L.N., Dirachka, A.I. and Sosin, V.A., Sov, Phys. Semicond. (USA), 16: 1646 (1982).

[9] Girlanda, R., Grasso, V. and Mondio, G., Solid Stat. Commun., 12: 179 (1973).

[10] Fisher, G. and Brebner, J.L., J. Phys. and Chem. Solids, 23: 1363 (1962).

[11] Gamal, G.A., Nassary, M.M., Hassein, S.A. and Nagat, A.T., Cryst. Res. Tech., 27: 629 (1992).

[12] Guder, H.S., Abay, B., Efeoglu, H. and Yogurtcu, Y.K., J. Luminescence, 93: 243 (2001).

[13] Abdulaev, G.B., Vodopyonov, L.K., Allakhverdiev, K.R., Golubev, L.V., Babaev, S.S. and Salaev, E.Yu., Solid State Commun., 31: 851 (1979).

[14] Milne, W.I. and Anderson, J.C., J. Phys. D. Appl. Phys., 7: 1540 (1974).

[15] Yamamoto, A., Syouji, A. and Goto,T., J. Luminescence, 87: 207 (2000).

[16] Abdul Rahman, M. and Belal, A.E., J. Phys. and Chem. Sol., 16: 925 (2000).

[17] Hussein, S.A. and Nagat, A.T., Cryst. Res. Technol., 24: 283 (1989).

[18] Nagat, A.T., Hussien, S.A., Gameel, Y.H. and Belal A.E., Egypt. J. Solids, 10: 45 (1988).

[19] Johnson, L. and Horovitz, K., Phys. Rev., 92: 226 (1953).

[20] Milne, W.I. and Anderson, J.C., J. Phys. D., 6: 2115 (1973). 


\section{القدرة الكهروحرارية للمركب التشالكو جينيدي الطبقي جاليو تيلرايد}

\section{أحمد عبد اللّه الغامدي}

قسم الفيزياء ، كلية العلوم ، جامعة الملك عبدالعزيز

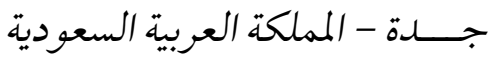

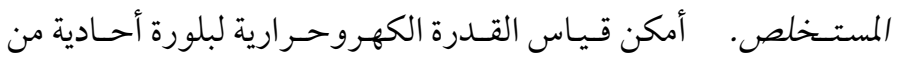

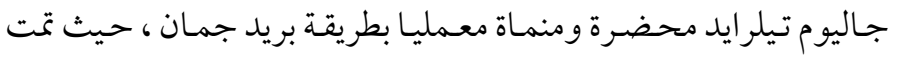

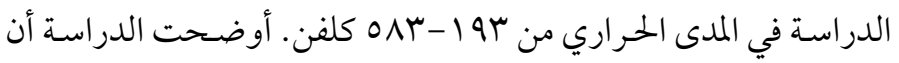

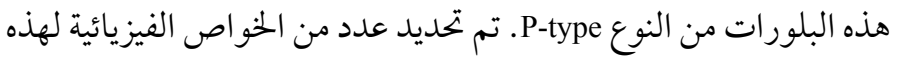

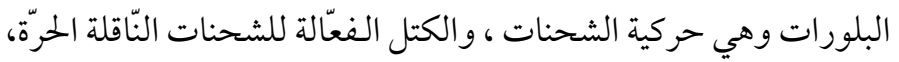

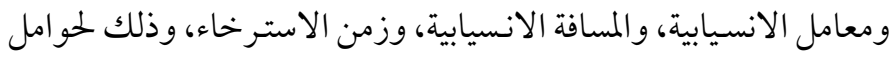

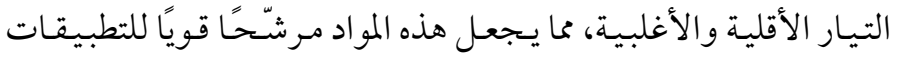
الحديثة بناء على خصائصها المميزّة. 